\title{
Estudo da ocorrência de enteroparasitas em hortaliças comercializadas na região metropolitana de São Paulo, SP, Brasil. I - Pesquisa de helmintos*
}

\author{
Study on the occurrence of intestinal parasites on vegetables commercially \\ traded in the metropolitan area of S.Paulo, SP, Brazil. I - Search for helminths
}

\author{
Carlos Augusto Fernandes de Oliveira ${ }^{\star \star}$, Pedro Manuel Leal Germano ${ }^{\star \star \star}$
}

\begin{abstract}
OLIVEIRA, C.A.F. de \& GERMANO, P.M.L. Estudo da ocorrência de enteroparasitas em hortaliças comercializadas na região metropolitana de São Paulo, SP, Brasil. I - Pesquisa de helmintos. Rev. Saúde públ., S.Paulo, 26: 283-89, 1992. Hortaliças in natura, comercializadas na região metropolitana de São Paulo, SP (Brasil), foram analisadas através de metodologia própria, visando à pesquisa e à identificação de formas de transmissão de helmintos intestinais de interesse médico. As hortaliças examinadas, constituídas de 50 amostras de cada variedade, foram de: alface (Lactuca sativa), variedades lisa e crespa, escarola (Chichorium sp) e agrião (Nasturtium officinale). Os resultados evidenciaram elevados percentuais de contaminação em todas as variedades analisadas, porém, as freqüências de helmintos foram maiores no agriāo. A escarola apresentou valores médios, geralmente situados entre as alfaces e o agrião. Os números médios de ovos e larvas por 100 gramas de amostra, embora elevados, não apresentaram diferenças estatisticamente significantes entre as quatro variedades de hortaliças estudadas. Uma grande variedade de helmintos, de ocorrência freqüente na população residente na Região Metropolitana de São Paulo, foi observada nas amostras. Os mais freqüentes, no entanto, foram: ancilostomídeos e Ascaris sp. Recuperaram-se também ovos de Toxocara sp, Fasciola sp e de tricostrongilídeos, comprovando a ocorrência de contaminação das hortaliças por fezes de animais domésticos. Considerando-se os resultados obtidos, ressalta-se a importância das hortaliças na transmissão de helmintíases intestinais, bem como a necessidade de medidas que propiciem uma melhoria na qualidade higiênico-sanitária destes alimentos.
\end{abstract}

Descritores: Contaminação de alimentos, análise. Helmintos, isolamento. Vegetais, parasitologia.

\section{Introdução}

As helmintoses intestinais apresentam distribuição mundial ${ }^{19} \mathrm{e}$, no Brasil, assumem papel relevante pelos elevados coeficientes de prevalência ${ }^{4,19}$, e pelas implicações clínicas e sociais que originam ${ }^{18,20}$.

No Estado de São Paulo, apesar do declínio das taxas de infecção de alguns enteroparasitas, as helmintoses intestinais ainda constituem sério problema de saúde pública, especialmente nos denominados cinturões de pobreza da região metropolitana da Capital ${ }^{28}$.

* Parte da Dissertação de Mestrado "Estudo da ocorrência de enteroparasitas em hortaliças comercializadas na regiảo metropolitana de São Paulo, SP - Brasil", apresentada à Faculdade de Saúde Priblica da USP, em 1991.

* Secretaria Municipal de Abastecimento de São Paulo São Paulo, SP - Brasil.

*** Departamento de Prática de Saúde Puiblica da Faculdade de Sáude Pública da USP - Sảo Paulo, SP - Brasil.

Separatas/Reprints: P.M.L.Germano - Av. Dr. Amaldo, 715 01246-904 - São Paulo, SP - Brasil.

Publicação financiada pela FAPESP. Processo Saúde Coletiva 91/4994-0
No país, freqüentemente as verduras são adubadas com dejetos humanos ou irrigadas com águas contaminadas com matéria fecal ${ }^{14,27}$. Soma-se a este fato o hábito alimentar de consumir hortaliças in natura, possibilitando a exposição de uma grande parcela da população às formas de transmissão desses parasitas ${ }^{14}$.

A verificação da presença de helmintos em hortaliças, portanto, reveste-se de grande interesse para a saúde pública, pois fornece dados para a vigilância sanitária sobre o estado higiênico desses produtos e permite o controle retrospectivo das condiçōes em que foram cultivados ${ }^{10,11}$.

As técnicas para exames parasitológicos de alimentos são, ainda, pouco desenvolvidas ${ }^{12,21}$. A maioria dos procedimentos visa à concentração de ovos e larvas nas amostras ${ }^{12}$, através de técnicas como a sedimentação espontânea ${ }^{11,13,16}$, centrifugação simples ${ }^{6,22,23}$, centrifugação simples associada à centrífugoflutuação $^{15}$ e ultracentrifugação ${ }^{14}$. Algumas técnicas incluem a incubação do sedimento para auxiliar na identificação e na verificação 
da viabilidade dos helmintos encontrados 22,23 . Isto porque, além da semelhança muito grande entre ovos e larvas de inúmeras espécies de parasitas do homem, de animais e mesmo de vegetais ${ }^{11}$, as hortaliças acumulam grãos de pólen, partículas vegetais e contaminantes do solo, dificultando a visualização e a identificação destas formas de transmissão.

No Brasil, não obstante a relevância e atualidade do problema, são poucos os trabalhos que estabelecem graus de contaminação de hortaliças por helmintos intestinais. Gelli e col. ${ }^{11}$ (1979), em 113 amostras de verduras comercializadas no Município de São Paulo, encontraram ovos e/ou larvas de ancilostomídeos $(59,3 \%)$ e de Strongyloides $s p$ $(5,3 \%)$. A contaminação por aqueles foi observada em $84,6 \%$ das amostras de rúcula, $78,3 \%$ de agrião, $53,3 \%$ de escarola e $32,4 \%$ de alface.

Hernandez e col. ${ }^{13}$ (1981) encontraram ovos de Ascaris $s p$ e de ancilostomídeos em hortaliças de 12 hortas do Município de Biritiba Mirim, SP, um dos principais produtores de verdura do Estado. Do mesmo modo, Motta e col. ${ }^{17}$ (1983), em Curitiba, e Miche \& Morganti ${ }^{16}$ (1983), em São Paulo, examinando, respectivamente 154 e 68 amostras de hortaliças detectaram, também, a ocorrência de elevados níveis de enteroparasitas nesses vegetais.

As hortaliças consumidas na Regiāo Metropolitana de São Paulo provêm, em grande parte, de pequenas propriedades localizadas nas proximidades da área urbana da Capital ou até mesmo a ela pertencente ${ }^{9}$, tendo-se registrado, em 1989 , um volume relativo de produção da ordem de $51,1 \%$ para alface, $72,5 \%$ para escarola e $93,5 \%$ para agriāo ${ }^{3}$. Esta área, denominada "cinturão verde", apresenta clima tropical temperado, com temperatura média anual de $25^{\circ} \mathrm{C}^{24}$. Nos meses de abril a setembro a irrigação artificial é utilizada com maior intensidade devido aos menores indices pluviométricos.

Com base nestes aspectos e considerando sobretudo a carência de informações sobre os níveis de contaminação desses produtos, constituem-se objetivos do presente trabalho:

- pesquisar qualitativa e quantitativamente a presença de formas de transmissão de helmintos de interesse médico em amostras de alface, variedades lisa e crespa, escarola e agriāo, provenientes de produtores do Estado de São Paulo e comercializadas na Companhia de Entrepostos e Armazéns Gerais de são Paulo (CEAGESP);

- estabelecer as possíveis diferenças entre os percentuais de contaminação dos quatro citados tipos de hortaliças.

\section{Material e Método}

\section{Amostra}

Foram utilizadas 50 amostras de cada tipo de hortaliça, alface (Lactuca sativa) variedades lisa e crespa, escarola (Chicorium $s p$ ) e agrião (Nasturtium officinale), considerando-se os elevados niveis de contaminação referidos por diversos autores do país ${ }^{11,13,14,16}$ e os estudos de Rude e col. ${ }^{22}$ (1984), perfazendo um total de 200 amostras. Estabeleceu-se como unidade amostral para as alfaces e escarolas, o pé (ou touceira) independentemente do peso ou tamanho que apresentavam, enquanto que para $o$ agrião considerou-se o maço, constituído de folhas agrupadas e seguras por um laço.

\section{Colheita de amostras}

Realizada diretamente junto à CEAGESP, adotou-se como critérios que cada amostra, independente de seu tipo, apresentasse boa qualidade e características organolépticas próprias, e representasse apenas um produtor do Estado de São Paulo.

A colheita de amostras compreendeu os meses de abril a outubro de 1990, época em que mais intensamente se utiliza a irrigação nas culturas de hortaliças ${ }^{24}$.

Assim, todas as segundas-feiras, no período da manhã, escolhia-se, aleatoriamente, 8 produtores retirando-se uma amostra do lote de hortaliças de cada um deles, de modo a obter-se duas amostras de cada tipo. Após a colheita, as amostras eram acondicionadas individualmente em sacos de polietileno estéreis, devidamente identificadas, e enviadas ao Laboratório de Controle de Alimentos da Prefeitura do Município de São Paulo, para análise. Este procedimento foi repetido semanalmente, até completar o número de amostras estabelecido.

\section{Análises das amostras}

No laboratório, utilizando-se luvas de borracha, as amostras eram pesadas, separando-se as folhas e desprezando-se aquelas manchadas ou deterioradas, bem como o talo. Em uma bandeja de aço inoxidável, colocava-se $300 \mathrm{ml}$ de solução de detergente neutro $(10 \mathrm{ml}$ de Extran MA $02^{R}$ diluídos em 2 litros de solução fisiológica) recém-preparada, mergulhando algumas folhas da amostra. Com auxilio de um pincel, esfregava-se toda a superfície das folhas, dcixando-se alguns segundos em repouso. Em seguida, 
as folhas eram levantadas para escorrer completamente o líquido, e depois desprezadas. Esta operação era repetida, com as demais folhas, até que toda a amostra fosse completamente lavada.

$O$ líquido obtido era filtrado, posteriormente, através de um funil analítico com gaze, e recolhido em um frasco cônico, onde era deixado em repouso por $24 \mathrm{~h}$. A bandeja era lavada duas vezes com $10 \mathrm{ml}$ de solução de detergente, recolhendo-se o líquido no mesmo frasco.

Completada a sedimentação, o líquido sobrenadante era desprezado cuidadosamente, transferindo-se os $30 \mathrm{ml}$ finais (contendo o sedimento), para um tubo de centrífuga de $50 \mathrm{ml}$. O cálice era lavado duas vezes com $10 \mathrm{ml}$ de solução fisiológica, recolhendo-se o líquido no mesmo tubo. A seguir, centrifugava-se o tubo a $2.600 \mathrm{rpm}$ durante um minuto, desprezando-se o sobrenadante.

O sedimento obtido era, então, ressuspendido com solução de Sulfato de Zinco (densidade igual a 1,200), centrifugando-se novamente a $2.000 \mathrm{rpm}$, durante um minuto. Após completar com solução de Sulfato de Zinco, gota a gota, até a altura da borda do tubo, transferia-se a película sobrenadante para um segundo tubo graduado de capacidade de $50 \mathrm{ml}$, acrescentando-se água destilada até 0,5 centímetros da borda do tubo. Procedia-se, novamente, à centrifugação a $2.600 \mathrm{rpm}$, durante $2 \mathrm{~min}$, desprezando-se o sobrenadante e ajustando-se o volume final de sedimento para $0,5 \mathrm{ml}$.

Após homogeneização do sedimento, com o auxílio de uma pipeta sorológica, transferia-se $0,05 \mathrm{ml}$ do volume final para uma lâmina de vidro, corando-se com solução de Lugol. A seguir a lâmina era coberta com lamínula para posterior exame ao microscópio, utilizando-se a objetiva $10 \mathrm{X}$ e percorrendo-se todo o campo para efetuar a identificação dos parasitas e a contagem dos ovos e larvas. A confirmação das estruturas foi realizada através da utilização da objetiva $40 \mathrm{X}$.

A identificação dos parasitas foi realizada até ao nível de gênero ou familia, conforme o tipo. Para auxiliar a identificação, realizou-se a mensuração, quando necessária, dos ovos ou larvas recuperados, adotando-se como referência os valores descritos por Burrows ${ }^{5}$ (1965) e Soulsby ${ }^{26}$ (1977).

\section{Cálculo do número de ovos e larvas}

Para o cálculo do número total de ovos e larvas na amostra utilizou-se a seguinte fórmula:

$$
\mathrm{N}_{\mathrm{A}}=\frac{\mathrm{a} \times 0,5}{0,05} \text {, onde }
$$

$\mathrm{N}_{\mathrm{A}}=\mathrm{n}^{\mathrm{0}}$ total de ovos e larvas na amostra $\mathrm{a}=\mathrm{n}^{\mathrm{O}}$ de ovos e larvas obtidos na lâmina $0,5=$ volume final do sedimento $(\mathrm{em} \mathrm{ml})$ $0,05=$ alíquota retirada $(\mathrm{em} \mathrm{ml})$

Com a obtenção desses valores, os resultados foram expressos em termos de número de ovos e larvas por 100 gramas de amostra, dado que:

$\mathrm{n}=\frac{\mathrm{N}_{\mathrm{A}}}{\mathrm{P}} \times 100 \mathrm{~g}$, onde

$\mathrm{n}=\mathrm{n}^{\mathrm{Q}}$ de ovos e larvas por $100 \mathrm{~g}$ de amostra $N_{A}=n^{9}$ total de ovos e larvas na amostra $\mathrm{P}=$ peso da amostra em gramas

\section{Análise Estatística}

Aos resultados obtidos no trabalho, foi aplicado o teste de duas proporçōes, com aproximação normal, para verificar a existência ou não de diferenças significantes entre os percentuais de contaminação, das quatro variedades de hortaliças analisadas, em relação às formas de transmissão de enteroparasitas encontrados.

Foi aplicada, ainda, a distribuição normal com o teste " $Z$ " para duas médias independentes, para determinar a existência ou não de diferenças significantes entre os números médios de ovos e larvas de helmintos recuperados nas amostras de hortaliças em estudo.

Adotou-se, como nível de rejeição, alfa igual a 0,05 e o valor crítico de " $Z$ " de alfa igual a 1,96 (Berquó e col.2 (1981)).

\section{Resultados}

Os níveis de contaminação das variedades de hortaliças amostradas (Tabela 1) variaram de $32,0 \%$, para alface lisa, a $66,0 \%$, nas amostras de agrião.

O teste de duas proporções revelou diferenças estatisticamente significantes entre os niveis de contaminação das amostras de agrião e alface lisa $(Z=3,40)$, entre agrião e alface crespa $(Z=3,20)$ e entre agrião e escarola $(Z=2,21)$.

A análise das freqüências de cada tipo de helmintos (Tabela 2) mostrou predominância da ocortência de ancilostomídeos, seguida de $A s c a-$ ris $s p$, em todas as variedades de hortaliças. Outros helmintos recuperados, com menor freqüência, incluíram Hymenolepis sp, Strongyloides $s p$, Trichocephalus $s p$, tricostrongilídeos, Taenia $s p$, Toxocara sp, Fasciola $s p$ e Enterobius $s p$. As 
Tabela 1 - Hortaliças amostradas, comercializadas na CEAGESP*, segundo as variedades de amostras $\theta 0$ resultado laboratorial da pesquisa de helmintos. São Paulo, 1991.

\begin{tabular}{|c|c|c|c|c|c|c|}
\hline \multicolumn{7}{|c|}{ Resultado Laboratorial } \\
\hline \multirow{2}{*}{$\begin{array}{c}\text { Variedade } \\
\text { de } \\
\text { amostra }\end{array}$} & \multicolumn{2}{|c|}{$P$} & \multicolumn{2}{|c|}{$\mathbf{N}$} & \multicolumn{2}{|c|}{ Total } \\
\hline & $\mathbf{F}$ & $\%$ & $\mathbf{F}$ & $\%$ & $\mathbf{F}$ & $\%$ \\
\hline Alface lisa & 16 & 32,0 & 24 & 68,0 & 50 & 100,0 \\
\hline Alface crespa & 17 & 34,0 & 33 & 66,0 & 50 & 100,0 \\
\hline Escarola & 22 & 44,0 & 28 & 56,0 & 50 & 100,0 \\
\hline Agriāo & 33 & 66,0 & 17 & 34,0 & 50 & 100,0 \\
\hline $\begin{array}{l}P=\text { Positivo } \\
N=\text { Negativo } \\
\text { CEAGESP } \\
\text { Armazéns Ger }\end{array}$ & & São & 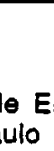 & $F=$ & $\begin{array}{l}\text { reqü } \\
\text { ercer }\end{array}$ & $\begin{array}{l}\text { ncia } \\
\text { tagem }\end{array}$ \\
\hline
\end{tabular}

amostras de agrião apresentaram, para todos os tipos de helmintos, as maiores freqüências de ocorrência, com exceção de tricostrongilídeos. $O$ menor nivel de contaminação foi observado nas amostras de alface lisa e crespa.

A aplicação do teste de duas proporções aos valores apresentados na Tabela 2 evidenciou diferenças estatisticamente significantes entre os percentuais de contaminação do agrião e da alface lisa, com relação aos ancilostomídeos $(\mathrm{Z}=2,08)$, Ascaris sp $(\mathrm{Z}=3,00)$, Hymenolepis $s p$ $(\mathrm{Z}=2,74)$ e Strongyloides sp $(\mathrm{Z}=2,00)$. As amostras de agrião apresentaram percentuais de contaminação significativamente superiores aos da alface crespa, com relação às freqüências de an- cilostomideos $(\mathrm{Z}=2,34)$ e de Ascaris $s p$ $(Z=3,00)$; e aos apresentados pela escarola, quanto às freqüências de Strongyloides $s p$ $(Z=2,45)$. Entre as amostras de escarola e de alface lisa, observou-se diferenças estatisticamente significantes apenas com relação às freqüências de Hymenolepis sp $(\mathrm{Z}=2,04)$.

Os valores médios de ovos e larvas de helmintos por $100 \mathrm{~g}$ de amostra, recuperados em cada tipo de hortaliça analisada (Tabela 3) variaram de $33,4 / 100 \mathrm{~g}$, para alface crespa, a $16,1 / 100 \mathrm{~g}$, para a escarola. A aplicação do teste de duas médias a estes valores revelou a existência de diferenças estatisticamente significantes apenas entre os números obtidos nas amostras de alface lisa e agrião $(Z=1,97)$.

\section{Discussão Conclusões}

Os resultados obtidos revelaram, em todas as variedades de hortaliças analisadas, elevados percentuais de contaminação por helmintos. Os valores encontrados refletem, igualmente, condiçōes e práticas de cultivo inadequadas do ponto de vista higiênico sanitário $10,11,22$.

Os resultados referem-se às análises efetuadas durante o período de menor pluviosidade nas áreas de cultivo de hortaliças ${ }^{24}$, época em que a irrigação das hortas é realizada com maior intensidade. Portanto, é possivel que os percentuais de contaminação nas épocas chuvosas apresentem valores sensivelmente mais baixos.

Tabela 2 - Hortaliças amostradas, comercializadas na CEAGESP*, segundo as variedades de amostras e os resultados laboratoriais da pesquisa de helmintos. São Paulo, 1991.

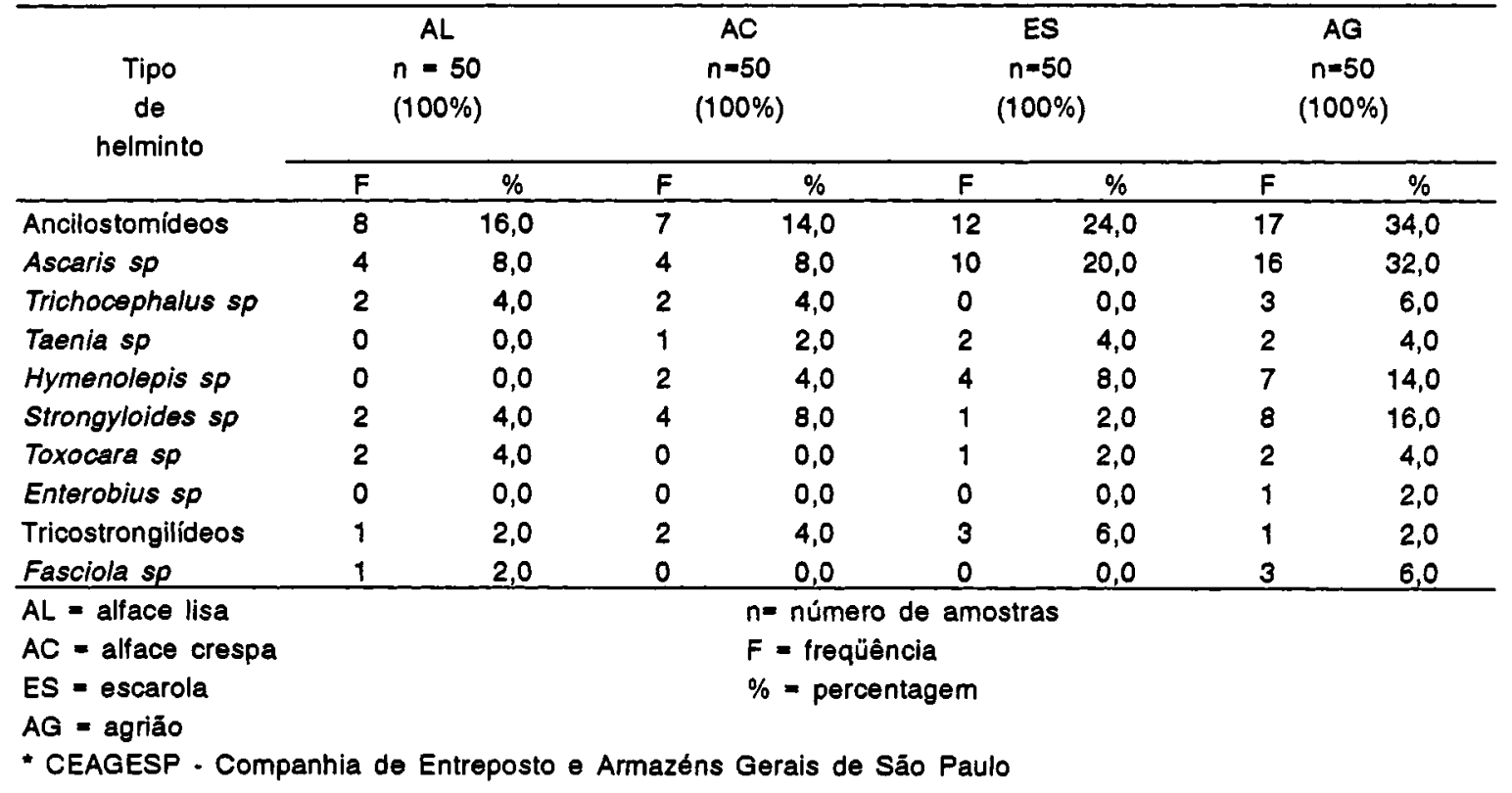


A metodologia analítica empregada, constituída por princípios de diversas técnicas consagradas em parasitologia clínica ${ }^{12}$, mostrou-se simples e de fácil execução, além de apresentar baixo custo e rapidez na obtenção de resultados.

As freqüências de helmintos maiores no agrião, seguidas da escarola, alface crespa e alface lisa são as mesmas das observadas por Gelli e col. ${ }^{11}$ (1979). Esses autores admitem que a estrutura do vegetal interfere com o grau de contaminação que o mesmo possa apresentar. Assim, o agrião, apresentando folhas múltiplas e separadas, com grande área de contato, permite maior fixação de enteroparasitas. A alface, por sua vez, apresenta folhas largas, firmemente justapostas, o que dificulta a aderência dos ovos e larvas. A escarola, possuindo características físicas intermediárias, apresenta níveis de contaminação situados entre o agrião e as alfaces lisa e crespa.

A estrutura física das hortaliças analisadas, certamente, contribui para a ocorrência de diferenças nos níveis de contaminação observados entre as quatro variedades. Isto se aplica, sobretudo, aos valores obtidos no agrião, que se mostraram significantemente maiores do que os encontrados nas outras hortaliças (Tabela 1).

Com relação às variações entre as freqüências específicas dos helmintos (Tabela 2), entretanto, este efeito parece ter sido discreto, uma vez que, para todas as comparações efetuadas, as diferenças mostraram-se estatisticamente significantes somente com relação a alguns dos tipos identificados (ancilostomídeos, Ascaris $s p$, Hymenolepis $s p$ e Strongyloides $s p$ ).

Embora os números médios de ovos e larvas por $100 \mathrm{~g}$ de amostra (Tabela 3 ) tenham sido superiores nas variedades de alface, não se observou, de maneira geral, diferenças estatisticamente significantes entre os tipos analisados. Esta constatação sugere uma interferência menor da estrutura das folhas no que concerne ao aspecto quantitativo da fixação de ovos ou larvas.
Esta é uma das razōes pela qual Choi e Sup Lee ${ }^{6}$ (1972) recomendam, também, a mensuração dos helmintos por unidade de peso, pois o tamanho e o volume das hortaliças é muito variável.

As diferenças entre os níveis de contaminação nas variedades de hortaliças estudadas, entretanto, parecem estar associadas, fundamentalmente, com as condições sanitárias do ambiente em que são cultivadas, diferentes em cada horta produtora, de acordo com as práticas de cultivo utilizadas $10,11,14$. Deste modo, os menores percentuais de contaminação, observados nas amostras de alface, podem ser atribuídos, entre outros fatores, às suas melhores condiçōes de cultivo, pois, segundo Christovão e col. ${ }^{7}$ (1967), estas hortaliças recebem cuidados mais adequados por parte dos horticultores. Diversos estudos comprovam que os ovos de helmintos podem sobreviver por períodos de tempo mais prolongados no meio aquático ${ }^{1,8}$. Este fato poderia justificar as freqüências superiores encontradas no agrião, cujo cultivo exige terrenos permanentemente úmidos, sobretudo daquelas relativas ao Hymenolepis sp, Enterobius sp e Strongyloides $s p$ (Tabela 2), cujas formas de transmissão são menos resistentes à dessecação ${ }^{1,8}$.

Com relação aos enteroparasitas recuperados, todos os tipos apresentam importância para a Saúde Pública 1,12,20. A maioria indica contaminação fecal de origem humana e/ou animal, tal como ocorre com Ascaris sp, Trichocephalus $s p$, Hymenolepis $s p$ e Strongyloides $s p$ e ancilostomídeos, uma vez que apresentam espécies de ocorrência no homem, nos animais ou em ambos 1,8,20. Há, portanto, similaridade entre estes resultados e os observados por outros autores, no pais $11,13,14,16,17$, embora as espécies de enteroparasitas referidas e suas respectivas freqüências tenham sido de menor valor.

Dentre os parasitas mais freqüentemente encontrados destacou-se o Ascaris sp (Tabela 2), cuja prevalência é bastante elevada na população

Tabela 3 - Hortaliças amostradas, comercializadas na CEAGESP*, segundo as variedades de amostras e os resultados da análise estatistica aplicada ao número de ovos e larvas obtidos na pesquisa quantitativa de helmintos. Sāo Paulo, 1991.

\begin{tabular}{lccccc}
\hline $\begin{array}{c}\text { Variedade } \\
\text { de } \\
\text { hortaliça }\end{array}$ & $\begin{array}{c}N^{2} \text { de } \\
\text { amostras } \\
\text { positivas }\end{array}$ & $\begin{array}{c}\text { Total de } \\
\text { ovos } \\
\text { larvas } \\
(/ 100 \mathrm{~g})\end{array}$ & $\begin{array}{c}\mathrm{N}^{2} \text { médio } \\
\text { de ovos } \\
\text { larvas } \\
(1100 \mathrm{~g})\end{array}$ & $\begin{array}{c}\text { Variân- } \\
\text { cia } \\
(100 \mathrm{~g})\end{array}$ & $\begin{array}{c}\text { Desvio } \\
\text { padrão } \\
(/ 100 \mathrm{~g})\end{array}$ \\
\hline Alface lisa & 16 & 501,3 & 31,3 & 606,1 & 24,6 \\
Alface crespa & 17 & 568,1 & 33,4 & $1,580,0$ & 39,7 \\
Escarola & 22 & 354,8 & 16,1 & 748,6 & 27,4 \\
Agrião & 33 & 546,9 & 16,6 & 548,3 & 24,2 \\
\hline
\end{tabular}

$N^{2}=$ Número

- CEAGESP - Companhia de Entreposto e Armazéns Gerais de São Paulo 
residente na Região Metropolitana de São Pau$10^{28}$, o que torna compatível o achado com a contaminação de origem humana. De modo inverso, as freqüências relativamente baixas de Strongyloides sp (Tabela 2), nas amostras de hortaliças estudadas, exceção feita ao agrião, podem ser devidas à reduzida prevalência deste helminto em nosso meio ${ }^{19,28}$.

Alguns autores consideram, no entanto, que a ordem de fregüuencia dos enteroparasitas nas hortaliças não é necessariamente a mesma encontrada na população humana do local estudado, devido sobretudo às diferenças na carga parasitária e na eliminação diária dos ovos pelos hospedeiros, variáveis para cada tipo de parasita $^{6,8}$. Feachem e col. ${ }^{8}$ (1983) estimam que em comunidades localizdas em países de clima tropical, em estágio de desenvolvimento, as concentrações de ovos de Trichocephalus $s p$ no esgoto, podem ser cinco vezes menores do que as de ovos de Ascaris sp. Esta relação, quando aplicada aos resultados obtidos no presente estudo, poderia explicar, entre outros fatores, as baixas freqüências encontradas para Trichocephalus $s p$ (Tabela 2), apesar da alta prevalência deste helmito na Região Metropolitana de São Paulo28.

A capacidade de sobrevivência de cada tipo de parasita no ambiente também deve ser considerada, pois as baixas freqüências obtidas para Hymenolepis $s p$ podem estar associadas a menor persistência dos ovos deste helminto no ambiente ${ }^{8}$.

As altas frequiências de ancilostomídeos, embora sejam concordantes com os resultados obtidos por Gelli e col." (1979), devem ser vistas com ressalvas, uma vez que os ovos de alguns parasitas de animais domésticos e de plantas, tais como Oesophagostomum sp e Meloidogyne $s p$, respectivamente, são muito semelhantes aos primeiros, dificultando a precisão diagnóstica destes helmintos $5,20,26$.

Outros parasitas recuperados, tais como Enterobius $s p$ e Taenia $s p$, são exclusivos do ho$\mathrm{mem}^{25}$, e fortalece a hipótese de ocorrência de contaminação por fezes humanas. Os ovos de Enterobius sp, além de pouco resistentes, geralmente não são eliminados nas fezes dos indivíduos parasitados 8,20 , o que justificaria a sua ocorrência rara nas amostras analisadas.

A freqüência de ovos de Taenia sp nas hortaliças examinadas mostrou-se relativamente baixa, embora a ocorrência das teníases na população da Região Metropolitana de São Paulo seja considerada endêmica ${ }^{20}$. É importante destacar que os ovos destes helmintos, além de serem eliminados pelo hospedeiros, em grande quantidade, apresentam resistência elevada, comparável a dos Ascaris $s p^{8,20,21}$. Por esta razão, os resultados obtidos confirmam a possibilidade de transmissão da cisticercose através das hortaliças, conforme já salientado por diversos autores $1,14,21$.

Ovos de Fasciola $s p$ e de tricostrongilídeos revelam contaminação por fezes de animais ruminantes 1,20 , utilizadas, provalvelmente, na adubação das hortas. Os ovos de Toxocara sp, por outro lado, indicam contaminação por fezes de canídeos e felídeos domésticos ${ }^{1,20}$.

A constatação da ocorrência de enteroparasitas em hortaliças, dada a magnitude dos resultados obtidos no presente estudo e a importância que apresenta para saúde pública, ressaltada a necessidade da adoção de medidas, por parte dos órgãos de vigilância sanitária, que propiciem uma melhoria da qualidade higiênica desses produtos.

OLIVEIRA, C.A.F. de \& GERMANO, P.M.L.

[Study on the occurrence of intestinal parasites on vegetables commercially traded in the metropolitan area of S. Paulo, SP, Brazil. I - Search for helminths]. Rev. Saúde públ., S. Paulo, 26: 283-89, 1992. Vegetables in natura, commercially traded in the metropolitan area of S. Paulo, were analysed by the appropriate methodology with a view to discovering an identifying the paths of transmission of enteroparasites of medical interest. The vegetables under study consisted of 50 samples of each variety listed below: lettuce (Lactuca sativa) oily leaves and crisphead varieties, endive (Chicorium sp) and water-cress (Nasturtium Officinale). Results showed high rates of contamination in all the vegetable varieties analysed. However, the water-cress was that which presented the highest frequencies of enteroparasites. The endive presented middle values ranking, in general, between the lettuces and the water-cress. Though high, the average number of helminth eggs and larvac obtained per $100 \mathrm{gr}$. of sample did not present statistically significant differences as between the four vegetable varietities studied. $A$ great variety of helminths and protozoans such as occur frequently in the resident population of the metropolitan area of S. Paulo, were observed in the samples. However, the most frequent were: hookworms and Ascaris sp. Eggs of Toxocara $s p$, Fasciola $s p$ and Trichostrongylidae were also recovered from the samples thus corroborating the occurrence of vegetables contamination with faeces of domestic animals. In view of the results obtained, the importance of these kinds of food in the transmission of enteroparasites is stressed, as well as the need for actions which improve the sanitary conditions of these products.

Keywords: Food contamination, analysis. Helminths, isolation and purification. Vegetables, analysis. 


\section{Referências Bibliográficas}

1. ACHA, P.N. \& SZYFRES, B. Zoonasis y enfermedades transmissibles comunes al hombre y a los animales. $2^{2}$ ed. Washington, D.C., Organización Panamericana de la Salud, 1986. (OPAS - Publicación Científica, 503)

2. BERQUO, E.S.; SOUZA, J.M.P.; GOTLIEB, S.L.D. Bioestatística. São Paulo, EPU, 1981.

3. BOLETIM ANUAL DA CEAGESP: 1989. São Paulo, Companhia de Entrepostos e Armazéns Gerais de São Paulo, 1989.

4. BOTERO, D. Persistencia de parasitoses intestinales endemicas en America Latina. Bol. Ofic. sanit. panamer., 90: 39-47, 1981.

5. BURROWS, RB. Microscopic diagnosis of the parasites of man. New Haven, Yale University Press, 1965.

6. CHOI, D.W. \& LEE,S. Incidence of parasites found on vegetables collected from markets and vegetable gardens in Taegu area. Korean J. Parasit., 10: 44-51, 1972.

7. CHRISTOVÃO, D.A.; IARIA, S.T.; CANDEIAS, J.A.N. Condições sanitárias das águas de irrigação de hortas do Município de São Paulo. 1 - Determinação da intensidade de poluição fecal através dos NMP de coliformes e de E. coli. Rev. Saúde públ., S. Paulo, 1: 3-11, 1967.

8. FEACHEM, R.G.; BRADLEY, D.J.; GARELICK, H.; MARA, D. Sanization and disease: health aspects of excreta and wastewater management. Chichester, John Wiley and Sons, 1983.

9. FILGUEIRA, F.A.R. Manual de olericultura: cultura $e$ comercialização de hortaliças. $2^{\natural}$ ed. São Paulo, Ed. Agronômica CERES, 1982. v.2.

10. GELDREICH, E.E. \& BORDNER, R.H. Fecal contamination of fruits and vegetables during cultivation and processing for market: a review. $J$. Milk Food Technol., 34: 184-95, 1971.

11. GELLI, D.S.; TACHIBANA, T; OLIVEIRA, I.R.; ZAMBONI, C.Q.; PACHECO, J.A.; SPITERI, N. Condições higiênico-sanitárias de hortaliças comercializadas na cidade de São Paulo, SP, Brasil. Rev. Inst. Adolfo Lutz S. Paulo, 39: 37-43, 1979.

12. HEALY, G.R.; JACKSON, G.J.; LICHTENFELS, J.R.; HOFFMAN, G.L.; CHENG, T.C. Foodbome parasites. In: Speck, M.L., ed. Compendium of methods for the microbiological examination of foods. $2^{\text {nd }}$ ed. Washington, D.C., American Public Health Association, 1984. p. 542-56.

13. HERNANDES, N.; CIMERMAN, B.; FERNANDES, M.F.P.; FERRAZ, C.A.M.; ARAUJO, A.L.; SILVA, C.A. Estudo da contaminação de verdura no Município de Biritiba Mirim. In: Congresso Brasileiro de Parasitologia, 6., Belo Horizonte, 1981. Resumos. Belo Horizonte, Sociedade Brasileira de Parasitologia, 1981. p. 219.

14. MARZOCHI, M.C.A. Estudo dos fatores envolvidos na disseminação dos enteroparasitas. II - Estudo da contaminação de verduras e solo de hortas na cidade de Ribeirão Preto, São Paulo, Brasil. Rev. Inst. Med trop. S. Paulo, 19: 148-55, 1977.

15. MASTRANDREA, G \& MICARELLI, A. Ricerca parassitaria nei prodotti vegetali prelevati da alcuni mercati rionali della cittá de Roma. Arch. ital. Sci. Med. trop. Parassit., 49: 55-9, 1968.
16. MICHE, M.P. \& MORGANTI, L.E. Parasitas em alfaces comercializadas na cidade de São Paulo. In: Congresso da Federación Latinoamericana de Parasitólogos, 6./ Congresso da Sociedade Brasileira de Parasitologia, 8./ Jomada Paulista de Parasitologia, 5. São Paulo, 1983. Resumos. São Paulo, Sociedade Brasileira de Parasitologia, 1983. p. 120.

17. MOTA, C.C.S.; ELIAS, A.; MIKOSZEWSKA, I.; VIEIRA, H.R.A.; PICHET NETO, J.; VASQUES, R.M.R.; ALMEIDA, A.A.; GAISSLER, M.S.; BEATRIZ, R; MOTA, R.M.T.C.S. Condiçōes higiênico-sanitátias de hortaliças comercializadas em Curitiba-PR (Brasil). In: Congresso Brasileiro de Ciência e Tecnologia de Alimentos, 6., Brasília, 1983. Programa e resumos. Brasilia, Sociedade Brasileira de Ciência e Tecnologia de Alimentos, 1983. p. 125.

18. NEGHME, A. \& SILVA, R. Ecologia del parasitismo en el hombre. Bol. Ofic. sanit. panamer, 70: 313-23, 1971.

19. ORGANIZACION MUNDIAL DE LA SALUD. Comite de Expertos en Prevención y Control de Infecciones Parasitarias Intestinales. Ginebra, 1986. Informe. Ginebra, 1987. (Serie de Informes Técnicos, 749)

20. PESSOA, S.B.\& MARTINS, A.V. Pessóa parasitologia médica. $1^{1}$ ed. Rio de Janeiro, Guanabara Koogan, 1982.

21. QUEVEDO, F. \& TAKUR, A.S. Parasitosis transmitidas por alimentos. Buenos Aires, Centro Panamericano de Zoonosis, 1980. (Serie de Monografias Científicas e Técnicas, C.P.Z., 12)

22. RUDE, R.A.; JACKSON, G.J.; BIER, J.W.; SAWIER, T.K.; RISTY, N.G. Survey of fresh vegetables for nematodes, amoebae and Salmonella. J. Assoc. off. anal. Chem., 67: 613-5, 1984.

23. RUDOLFS, W.; FALK, L.L.; RAZOTZKIE, RA. Contamination of vegetables grow in pollued soil. III - Field studies on Ascaris eggs. Sew. ind Wastes, 23: 656-60, 1951.

24. SECRETARIA DE ECONOMIA E PLANEJAMENTO. GEGRAN. Diagnóstico da primeira Regiāo Administrativa do Estado de São Paulo. São Paulo, 1973.

25. SHUVAL, H.I.; YEKUTIEL, P.; FATTAL, B. Epidemiological evidence for helminth and cholera transmission by vegetables irrigated with wastewater: Jerusalem - a case study. Wat. Sci Technol., 17: 433-42, 1984.

26. SOULSBY, E.J.L. Helminths, arthropods and protozoa of domesticated animals (Mönnig's). $6^{\text {th }} \mathrm{ed}$. Philadelphia, Lea \& Febiger, 1977.

27. VALLADA, E.P.; SEIXAS, A.S.S.; CARRARO, K.M.A. Contribuição ao estudo da transmissão das enteroparasitoses. Rev. bras. Farm., 68: 102-8, 1987.

28. WALDMAN, E.A. \& CHIEFFI, P.P. Enteroparasitoses no Estado de São Paulo: questão de saúde pública. Rev. Inst. Adolfo Lutzo S. Paulo, 49: 93-9, 1989.

Recebido para publicação em 17/12/1991 Reapresentado em 28/2/1992 Aprovado para publicaçāo em 4/3/1992 